\title{
Radical Mediated Thiol-Ene/Yne Dispersion Polymerizations
}

\author{
Farbod Alimohammadi, ${ }^{\text {a) }}$ Chen Wang, ${ }^{\text {b) }}$ Olivia Z. Durham, ${ }^{\text {a) }}$ Hannah R. Norton, ${ }^{\text {a) }}$ \\ Christopher N. Bowman, ${ }^{\text {b), * Devon A. Shipp }}{ }^{\text {a), * }}$ \\ a) Department of Chemistry and Biomolecular Science \\ and Center for Advanced Material Processing \\ Clarkson University, Potsdam, NY 13699-5810 \\ b) Department of Chemical and Biological Engineering \\ University of Colorado, UCB 596, Boulder, CO 80303 \\ * To whom correspondence should be addressed \\ Christopher.bowman@colorado.edu
}

dshipp@clarkson.edu

\begin{abstract}
We report the synthesis of polymeric particles using radical mediated step-growth thiolene and thiol-yne 'click' dispersion polymerizations in alcoholic solvents. Various alkene, alkyne and thiol monomers were used, and thermal, photo- or redox initiation methods were all shown to be effective means to initiate polymerization. Polymer particles typically were formed with diameters in the range of several hundred nanometers, with narrow size distributions though broader than typical free-radical chain-growth dispersion polymerizations. Photoinitiation yielded the smallest sizes due to the rapid nucleation of particles as compared to redox and thermal initiation methods. Reaction kinetics were monitored by FT-IR spectroscopy for aliquot samples taken at various reaction durations. The reaction achieved full conversion in
\end{abstract}


photoinitiated systems within five minutes, while it took three hours for both thermal and redox initiation to be completed. The effects of polymerization conditions on particle size particularly the impact of monomer, stabilizer, and initiator concentrations were studied. Generally, average particle size increased with higher monomer concentration and decreased with additional stabilizer or initiator. Combinations of monomers with varying number of functional groups were investigated to form particles of various mechanical and physical behavior, including both linear and crosslinked systems. 


\section{Introduction}

The production of polymeric colloids by thiol-ene, thiol-yne and thiol-Michael polymerizations has recently gained significant interest because of their many inherent benefits [1-14]. The radical-mediated thiol-ene/yne polymerizations embody multiple desirable attributes associated with 'click' chemistry principles, such as having readily available monomers, achieving excellent yields and selectivity, and having high atom efficiency and reaction orthogonality [15-19]. These polymerizations occur in a step-growth manner [20] which enables post-polymerization functionalization simply by altering the reaction stoichiometry, and it is readily possible to alter the backbone structure of the network simply by changing the monomer cores. Additionally, there are multiple radical initiation modes available, including photo, thermal, and redox systems. Thus appropriate reaction conditions are readily chosen, particularly under circumstances in which the presence of sensitive functional groups necessitates solvent, temperature or other environmental conditions. The formation of polymer colloids using 'click' thiol-ene/yne reactions has the potential to significantly impact polymeric particle technologies, with the combination of flexibility, functionality, and excellent controllability achievable by these reactions.

Previously reported thiol-X colloidal preparative systems include suspension thiolene/yne radical polymerizations [1-4], miniemulsion thiol-ene/yne radical polymerizations $[1,8,10-14]$ microfluidic thiol-ene/yne and thiol-isocyanate polymerizations $[9,21]$ and thiolMichael addition dispersion polymerization [5-7,22]. Each of these systems result in a polymer dispersed phase and either a water continuous phase (suspensions and miniemulsions) or an alcohol continuous phase (dispersions); typically the former two begin as two phase systems, while dispersion polymerizations begin as a homogeneous solution of monomer and the polymer 
phase separates during the polymerization process [23]. While other step-growth polymerizations have been used to make polymer colloids [24,25], including polyurethanes [26], epoxy resins [27], polyesters [28], and polyolefins in miniemulsions [29-33], the robustness and flexibility of the 'click' thiol-ene/yne approach provides significant advantages. For example, thiol-ene/yne polymerizations allow easy particle functionalization, controlled porosity, degradability and facilitate the use of these particles in imaging applications.

However, to the best of our knowledge radical thiol-ene/yne dispersion polymerizations have not been studied. Radical mediated reactions are particularly advantageous in a wide selection of monomers, multiple initiation modes and tolerance in acidic, basic and neutral conditions. Furthermore, radical dispersion polymerizations typically are simple, high yielding and require lower homogenization energies than emulsion and suspension polymerizations. Herein, we study multiple initiation modes in thiol-ene dispersion polymerizations, as well as the effects of polymerization conditions on particle size, including reactive species concentrations, stabilizers and monomer types.

\section{Experimental}

Materials. 1-Hydroxycyclohexyl phenyl ketone, 2,2' -azobis(2-methylpropionitrile) (AIBN), ethyl-4-(dimethylamino)benzoate, benzoyl peroxide, 1,3,5-triallyl-1,3,5-triazine2,4,6(1H,3H,5H)-trione (TTT), pentaerythritol tetrakis (3-mercaptopropionate) (PETMP), trimethylolpropane diallyl ether (TMP), trimethylolpropane tris(3-mercaptopropionate) (TMTMP), 1,6-hexanedithiol (HDT), 5-hexynoic acid (HYA), tris[2-(3mercaptopropionyloxy)ethyl] isocyanurate (TMPEIC) and 1,7-octadiyne (ODY) were all obtained from Sigma Aldrich. Structures of these monomers are shown in Scheme 1. Ethyl 
acetate, methanol and acetone were purchased from J.T. Baker, and methanol from Fisher Scientific. Poly $(N$-vinylpyrrolidone) (molecular weight,55,000 g/mol) was obtained from Sigma Aldrich. All chemicals were used as received.

Instrumentation. Nuclear magnetic resonance (NMR) spectroscopy was conducted using a Bruker Avance DMX-400 instrument. FT-IR data were collected from a Bruker Vector 22 FTIR spectrometer, except for redox-initiated polymerizations, which were obtained from a Thermo Nicolet 6700 with a diffuse reflectance setup. Dried microparticles were well mixed with $\mathrm{KBr}$ powder in a mortar before FT-IR analysis. Field emission scanning electron microscopy (FESEM) was performed on a JEOL JSM 6300 instrument. For electron microscope analysis, drops of particle dispersion were placed on an aluminum stub and subsequently dried in a vacuum oven. Prior to analysis, samples were sputter-coated with approximately $6 \mathrm{~nm}$ thick $\mathrm{Au} / \mathrm{Pd}$ layer. Particle size distributions in the dispersions were measured on a ZetaPALS instrument (Brookhaven Instruments).

Procedures. All reactions consisted of $10 \mathrm{~g}$ methanol solution, and had a 1:1 molar ratio of alkene and thiol functional groups from the monomers. A small amount of co-solvent (ethyl acetate or acetone, 1:1 volume ratio relative to the monomers) was added to dissolve the monomers. Initiators were added to the mixture and dissolved before curing (photo-, thermal or redox). Reactions were carried out with magnetic stirring in a $20 \mathrm{ml}$ glass vial with a Teflon coated micro stir bar. Photopolymerizations were initiated using 1-hydroxycyclohexyl phenyl ketone (amounts are given in Table 1) as a photoinitiator, with 5 minutes exposure to UV light (365 nm, $11 \mathrm{~mW} / \mathrm{cm}^{2}, \sim 20 \mathrm{~cm}$ away from the lamp). Thermal polymerizations were initiated using AIBN (amounts are given in Table 2), and the reaction was carried out at $60^{\circ} \mathrm{C}$ for 3 hours. Redox-initiated polymerizations were initiated by equal molar ethyl-4-(dimethylamino)benzoate 
and benzoyl peroxide (amounts are given in Table 3). Initiators were added separately from their stock solutions in methanol, and the mixture was stirred at ambient conditions overnight before characterizations.

Kinetic measurements for redox-initiated polymerization. Redox-initiated PETMP-TTT dispersion polymerizations were carried out with $10 \mathrm{wt} . \%$ initiator with respect to monomers, and 2 wt.\% monomer loading with respect to solvents $\mathrm{In}$ a $50 \mathrm{~mL}$ round bottom flask, a clear solution consisting of $0.37 \mathrm{~g}$ PETMP, $0.25 \mathrm{~g}$ TTT, $0.034 \mathrm{~g}$ BPO and $0.3 \mathrm{~g}$ PVP in a mixture of $38.7 \mathrm{~mL}$ methanol and $0.5 \mathrm{~mL}$ ethyl acetate was prepared. The mixture was purged with argon for $30 \mathrm{~min}$ and a $0.1 \mathrm{~mL}$ solution of $0.028 \mathrm{~g}$ EDAB in methanol was injected to initiate the polymerization. Small aliquots were withdrawn from the reaction mixture by an argon-purged syringe. Each aliquot was immediately exposed to air and an FT-IR spectrum was subsequently taken. The peak area between $3000-2800 \mathrm{~cm}^{-1}$ was used as internal standard, and the peak centered at $2570 \mathrm{~cm}^{-1}$ (representing SH-stretching vibrations) was used to determine the conversion. 
<smiles>O=C(CCS)OCC(COC(=O)CCS)(COC(=O)CCS)COC(=O)CCS</smiles><smiles>C=CCn1c(=O)n(CC=C)c(=O)n(CC=C)c1=O</smiles><smiles>CCC(COC(=O)CCS)(COC(=O)CCS)COC(=O)CCS</smiles><smiles>C=CCOCC(CC)(CO)COCC=C</smiles><smiles>C#CCCCCC(=O)O</smiles>

Chart 1. Structures of various monomers used in this work; Multi-thiol monomers: pentaerythritol tetrakis (3-mercaptopropionate) (PETMP), trimethylolpropane tris(3mercaptopropionate) (TMTMP), 1,6-hexanedithiol (HDT) and tris[2-(3mercaptopropionyloxy)ethyl] isocyanurate (TMPEIC); Multi-alkene/alkyne monomers: 1,3,5triallyl-1,3,5-triazine-2,4,6(1H,3H,5H)-trione (TTT), trimethylolpropane diallyl ether (TMP), 5hexynoic acid (HYA) and 1,7-octadiyne (ODY). 


\section{Results and Discussion}

\section{Various Initiation Modes}

Firstly, we sought to test thermal, photo and redox initiation modes in thiol-ene dispersion polymerizations. A $1 \mathrm{wt} . \%$ solution of equimolar ene:thiol functionality of TTT (triene) and PETMP (tetra-thiol) in methanol was prepared, along with $50 \mathrm{wt} . \%$ (relative to monomers) PVP and a particular initiator. A small amount of ethyl acetate was added to completely dissolve PETMP, and the reaction mixture was kept under magnetic stirring. In all three cases, the homogeneous solution became cloudy and opaque, indicating the precipitation of polymer chains, similarly to traditional chain-growth dispersion polymerizations such as polystyrene. However, the precipitation time varied in those initiation modes. In photoinitiated polymerizations it took 2-3 minutes to precipitate after the exposure, while it took $\sim 20-30$ minutes for the redox-initiation system and $\sim 30-45$ minutes for the thermally initiated systems, depending on the polymerization conditions.

Figure 1 shows SEM images and particle size distributions of the microparticles made from stoichiometric PETMP and TTT. Clearly, each approach yielded spherical (or nearly spherical) particles, with diameters ranging from a few hundred nanometers up to micrometers. Particularly, thermal initiation with $1 \mathrm{wt} . \%$ monomer in methanol resulted in submicron particles with uneven surfaces (Figure 1a-b), which was possibly due to the fact that the particle was growing at a temperature above its glass transition temperature. Dynamic light scattering (DLS) measured the average particle diameter as $391 \mathrm{~nm}$ with a polydispersity of 0.126 (Figure 1c), which was a wider distribution than typically found in dispersion polymerization systems. For photoinitiation with 1 wt.\% monomer in methanol, spherical particles with an average diameter of $191 \mathrm{~nm}$ (Figure 1d-e) and a polydispersity of 0.194 (Figure 1f) were prepared. For redox 
initiation, spherical particles were observed (Figure 1g-h), and the DLS measurement indicated an average diameter of $351 \mathrm{~nm}$ and a polydispersity of 0.056 (Figure 1i). Norrish type-I photoinitiators (aromatic ketones, for example) are well known to generate free radicals much faster than thermal initiators (azo-type, for example) with appropriate irradiation conditions. Previous reports of step-growth dispersion polymerizations show that the particle size is strongly dependent on reaction kinetics, and a faster reaction leads to smaller particles [7]. The fact that under similar conditions photoinitiation produced the smallest particles is thus in agreement with previous studies. Particle size distributions are not particularly narrow, indicating that the particle nucleation is not fast relative to particle growth. Particularly, thermal and photo initiation both generated less uniform particles than that found for redox initiation. On one hand, the nonuniformity in thermal initiation is possibly due to the aggregation between growing nuclei, which is likely to happen when the material is heated above its $\mathrm{Tg}$ as the chain mobility is maximized. On the other hand, the wide distribution from photoinitiation is potentially due to light attenuation in opaque colloidal systems, which causes heterogeneity in the reaction rate throughout the vessel. The dispersions remained colloidally stable over several weeks, and samples with smaller particles were stable for even longer. 


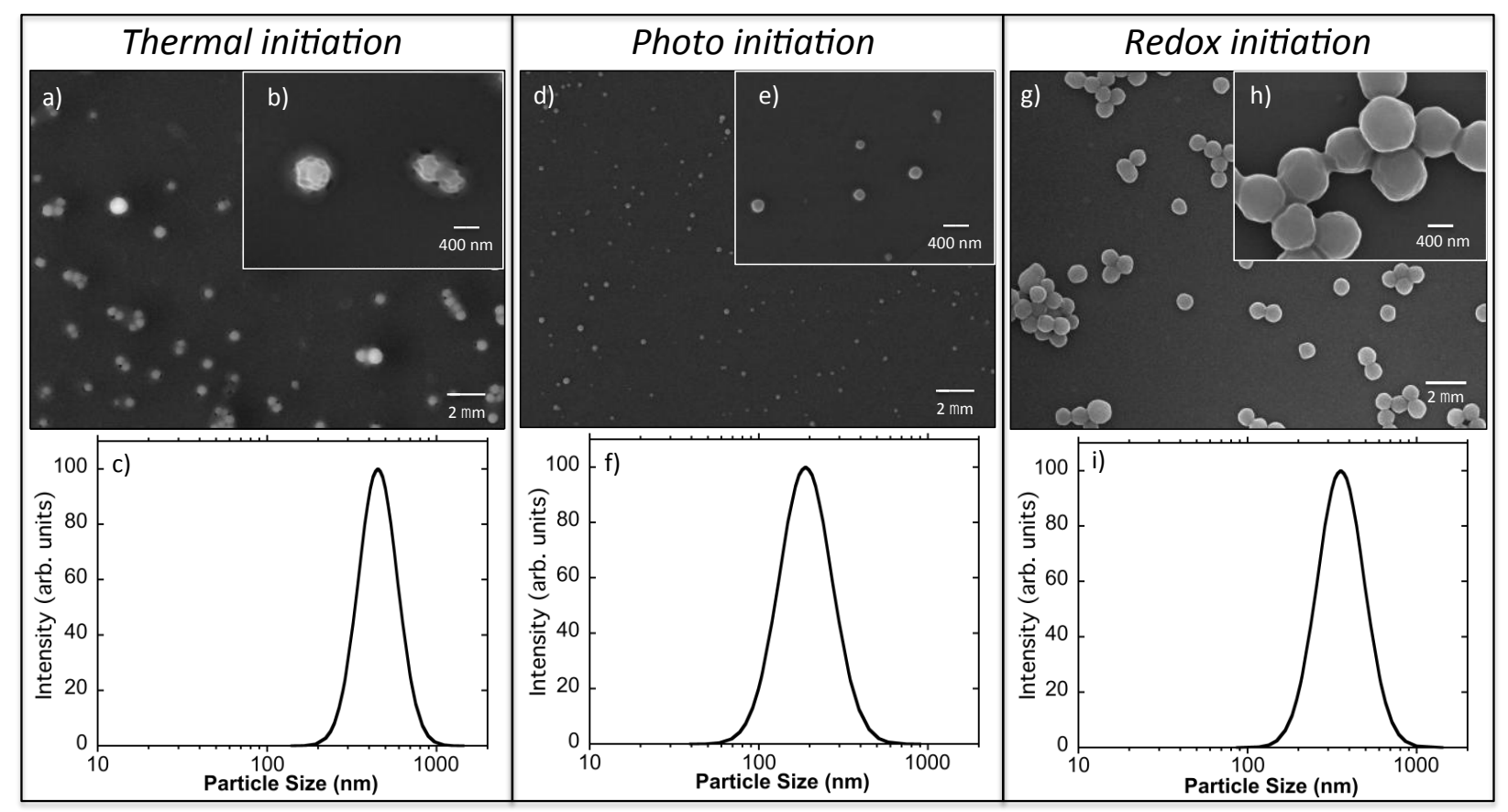

Figure 1. SEM and DLS curves for $(a-c)$ thermally-, $(d-f)$ photo-, and $(g-i)$ redox-initiated radical thiol-ene dispersion polymerizations. Thermal initiation (TI-3): 0.04g (0.035 ml) TTT, 0.06g (0.047 ml PETMP), $10 \mathrm{~g}$ methanol, $0.01 \mathrm{~g}$ AIBN, $0.073 \mathrm{~g}(0.082 \mathrm{ml})$ ethyl acetate, $0.05 \mathrm{~g}$ PVP. $60^{\circ} \mathrm{C}$ for 3 hours; DLS data: Avge. diameter $=391 \mathrm{~nm}$, polydispersity $=0.126$. Photo initiation (PI-3): $0.04 \mathrm{~g}(0.035 \mathrm{ml}) \mathrm{TTT}, 0.06 \mathrm{~g}$ (0.047 ml PETMP), $10 \mathrm{~g}$ methanol, $0.01 \mathrm{~g}$ initiator (1-hydroxcyclohexyl phenyl ketone), $0.073 \mathrm{~g}$ (0.082 ml) ethyl acetate, $0.05 \mathrm{~g}$ PVP; DLS data: Avge. diameter $=194 \mathrm{~nm}$, polydispersity $=0.194$. Redox initiation $(R-4):$ Avge. diameter $=$ $351 \mathrm{~nm}$, polydispersity $=0.056$.

\section{Polymerization Kinetics}

To determine the polymerization kinetics for the redox and thermal systems, we followed the loss of the ene and thiol functionality using FT-IR at various reaction times. Photo-initiated polymerizations occurred much more rapidly (within $\sim 5$ mins) and reached full conversion within approximately 5 minutes of irradiation (see FT-IR spectrum of final product in Figure SI- 
1, Supporting Information). Figure 2 shows the FT-IR spectra collected from the redox-initiated polymerization. At various reaction times from the beginning to three hours, spectra were similar except for the decrease in peaks centered at $3085 \mathrm{~cm}^{-1}$ and $2570 \mathrm{~cm}^{-1}$, which represented alkene and thiol groups, respectively. Note that the signals for both groups reduce at the same rate, indicative of the 1:1 stoichiometry of the thiol-ene reaction. Figure 3 shows the thiol functional group conversion as a function of time for both the redox- and thermally-initiated polymerizations (see FT-IR spectrum of final product from thermal initiation in Figure SI-1, Supporting Information). The peak area between $3000-2800 \mathrm{~cm}^{-1}$ was used as an internal standard to eliminate possible variances of IR absorption among samples. Close to $100 \%$ conversion was reached within three hours in both cases, which confirmed the reaction efficiency of the thiol-ene "click" reaction in dispersion systems. Further, the kinetics for these reactions are readily altered to a preferred timeframe, by simply changing the initiator concentration, temperature or light intensity, depending on the system being used.

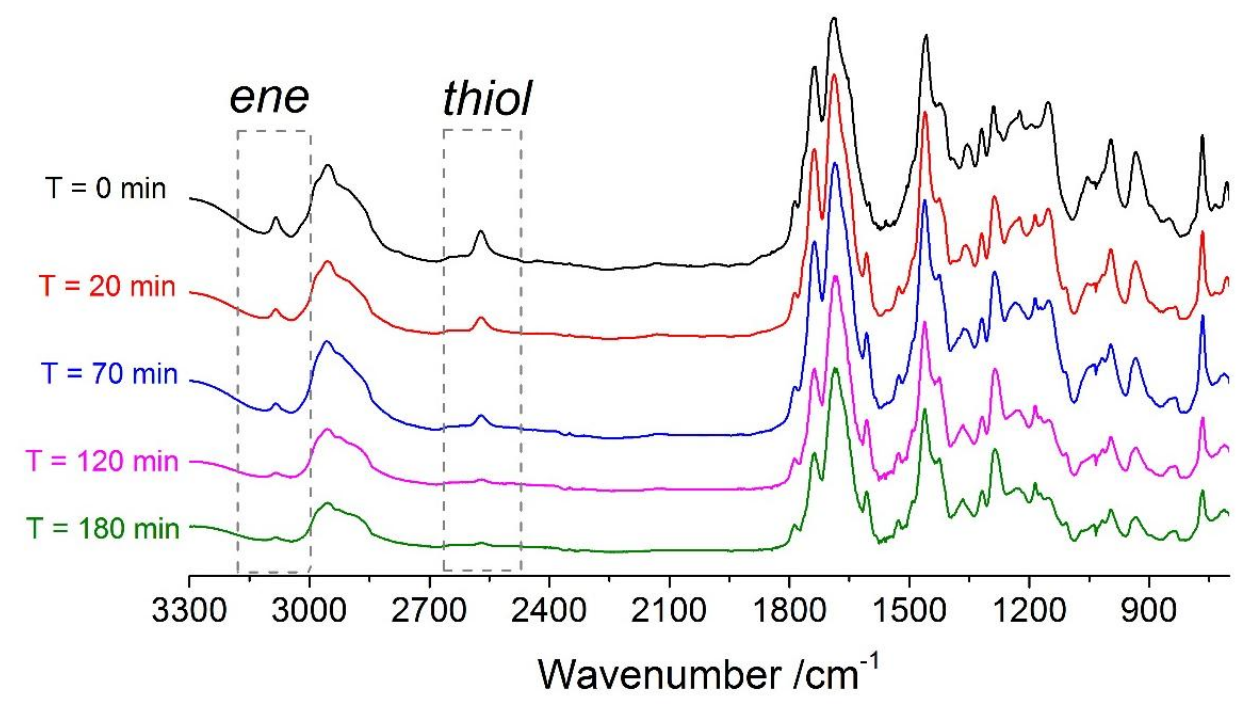

Figure 2. FT-IR spectra for redox-initiated PETMP-TTT dispersion polymerization at various reaction times. Reaction conditions: stoichiometric mixture of PETMP-TTT in methanol (2 wt.\%); equal molar BPO and EDAB (10 wt.\% in total with respect to monomers). 


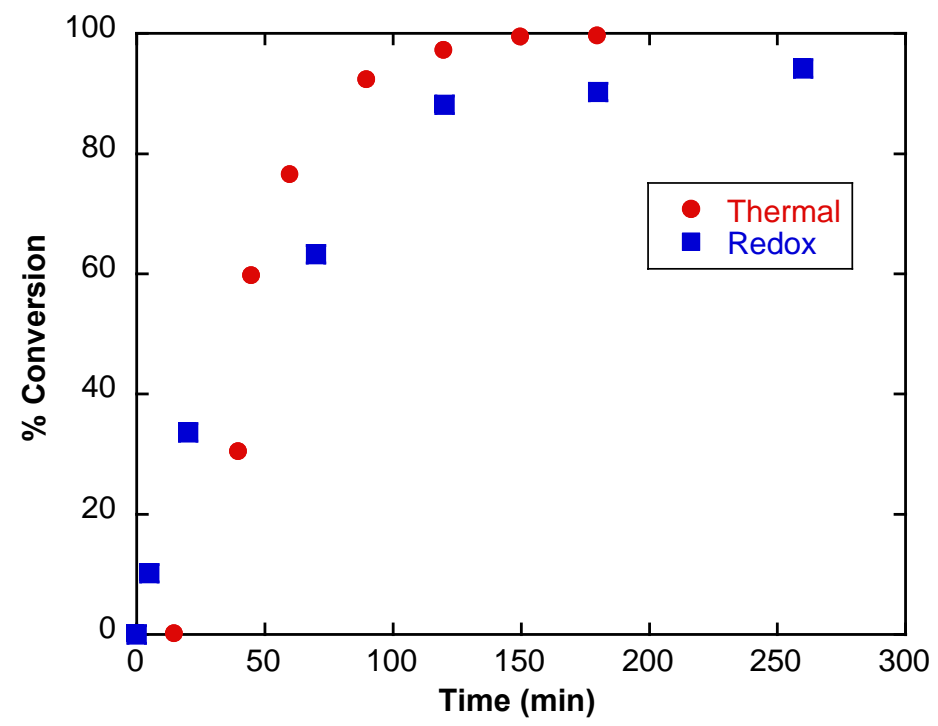

Figure 3. Reaction conversion profiles for stoichiometric PETMP-TTT dispersion polymerizations initiated by redox and thermal systems. Redox reaction conditions: stoichiometric mixture of PETMP-TTT in methanol (2 wt.\% with respect to solvent); equal molar BPO and EDAB (10 wt.\% with respect to monomers). Thermal reaction conditions: stoichiometric mixture of PETMP-TTT ( $1 \mathrm{~g}$ combined in $10 \mathrm{~g}$ methanol) and AIBN (0.002 $\mathrm{g}$ ) at $60^{\circ} \mathrm{C}$.

Effect of Various Parameters on Particle Size and Distribution: Photo-Initiation

Table 1 summarizes the reactions that were performed using photo-initiation with various organic (monomer) contents, initiator concentrations, stabilizer concentrations, and co-solvent types. Reactions PI-1 through PI-5 have increasing amounts of monomer from $0.5 \mathrm{wt} . \%$ to $3 \mathrm{wt}$ $\%$ in methanol, with the same amount of initiator and stabilizer with respect to monomers. Increasing monomer concentration led to a consistent increase in particle size. Increasing the monomer content only (PI-6 - PI-9; i.e., maintaining the initiator and stabilizer content) resulted 
in an increase in particle size that was similar to the PI-1 - PI-5 reaction series. It is worth noting that the particle diameters for PI-6 - PI-9 series were slightly different from those for the PI-1 PI-5 series, possibly due to the variance in the initiator and stabilizer amounts. To evaluate the effect of stabilizer, the PI-10 - PI-13 series was carried out with variance exclusively in the amount of PVP that was used. Surprisingly, particles were successfully prepared with no stabilizer present (PI-10). In classic chain-growth dispersion polymerizations, for example polystyrene, the presence of stabilizer is mandatory to prevent aggregation of growing particles, which is caused by the inevitable Brownian motion.[34] Our observations here may be the result of such short reaction times (less than 5 mins) where the probability of covalent linkages between particles is limited, and/or the crosslinked PETMP/TTT system significantly lowers the mobility of the polymer chains compared to the typically non-crosslinked polymers made by chain-growth dispersion polymerization. As the PVP concentration increased, the particle size decreased significantly from $208 \mathrm{~nm}$ to $158 \mathrm{~nm}$ (PI-11 - PI-13 and PI-3), which is in good agreement with many other dispersion polymerization systems. Acetone and ethyl acetate worked similarly as co-solvents and had a negligible effect on particle size, as the co-solvent was used in a very small fraction so that it has an insignificant effect on the solubility/polarity. (PI-3, PI-14). Further, smaller particles were prepared with an increased initiator amount (PI-3, PI-15 PI-17), which followed the previously discussed kinetics effect on particle sizes. 
Table 1. Summary of Photoinitiated Polymerizations. All reactions used $10 \mathrm{~g}$ methanol, PVP as stabilizer, 1-hydroxycyclohexyl phenyl ketone as initiator, 1:1 mole ratio of monomers (PETMP + TTT), irradiated for 5 minutes, and 1:1 (vol.) of co-solvent (ethyl acetate, unless otherwise stated) relative to the monomers. PI-3 is a reference reaction.

\begin{tabular}{|c|c|c|c|c|c|}
\hline \multirow{2}{*}{$\begin{array}{l}\text { Reaction } \\
\text { Name }\end{array}$} & \multicolumn{3}{|c|}{ Reactant Amounts (g) } & \multirow{2}{*}{$\begin{array}{l}\text { Avg. Particle } \\
\text { Diameter } \\
(\mathrm{nm})\end{array}$} & \multirow[t]{2}{*}{ Polydispersity } \\
\hline & Monomers & Initiator & $\begin{array}{l}\text { Stabilizer } \\
\text { (PVP) }\end{array}$ & & \\
\hline PI-1 & 0.05 & 0.005 & 0.025 & 160 & 0.243 \\
\hline PI-2 & 0.072 & 0.0071 & 0.0357 & 178 & 0.195 \\
\hline PI-3 & 0.10 & 0.01 & 0.05 & 194 & 0.194 \\
\hline PI-4 & 0.20 & 0.02 & 0.10 & 273 & 0.165 \\
\hline PI-5 & 0.30 & 0.03 & 0.15 & 332 & 0.154 \\
\hline PI-6 & 0.05 & 0.01 & 0.05 & 162 & 0.150 \\
\hline PI-7 & 0.072 & 0.01 & 0.05 & 177 & 0.164 \\
\hline PI-3 & 0.10 & 0.01 & 0.05 & 194 & 0.194 \\
\hline PI-8 & 0.20 & 0.01 & 0.05 & 222 & 0.203 \\
\hline PI-9 & 0.30 & 0.01 & 0.05 & 309 & 0.136 \\
\hline PI-10 & 0.10 & 0.01 & 0 & 208 & 0.162 \\
\hline PI-11 & 0.10 & 0.01 & 0.025 & 188 & 0.193 \\
\hline PI-12 & 0.10 & 0.01 & 0.10 & 178 & 0.184 \\
\hline PI-13 & 0.10 & 0.01 & 0.15 & 158 & 0.227 \\
\hline PI-3 & 0.10 & 0.01 & 0.05 & 194 & 0.194 \\
\hline PI-14 & 0.10 & 0.01 & 0.05 (acetone) & 201 & 0.203 \\
\hline
\end{tabular}




\begin{tabular}{llllll}
\hline PI-15 & 0.10 & 0.05 & 0.05 & 176 & 0.182 \\
PI-16 & 0.10 & 0.10 & 0.05 & 180 & 0.201 \\
PI-17 & 0.10 & 0.20 & 0.05 & 154 & 0.233 \\
\hline
\end{tabular}

Effect of Various Parameters on Particle Size and Distribution: Thermal Initiation

Data collected from thermally initiated polymerizations are provided in Table 2; here we studied the effect of species concentrations in a similar manner to the photoinitiated reactions. Overall, we observed that the thermal polymerizations yielded larger particles than those formed by the photoinitiated systems. This outcome may be due to a more extended nucleation stage, which leads to fewer nuclei as a result of nuclei aggregation in the slower thermally initiated polymerizations. The trend on monomer/stabilizer concentration was similar to the photoinitiated system. In particular, the particle size increased when either more monomer and/or less stabilizer was used. However, we noticed an upper limit for monomer concentration, as aggregation occurred for $3 \mathrm{wt} . \%$ loading of monomers (TI-9, Table 2). Also, particles could not be formed without stabilizer (TI-10). Increasing the initiator concentration firstly seems to reduce the particle size (TI-15 - TI-17, TI-3), but at the highest concentration the particle size increased slightly, although this might be within experimental reproducibility. Again, ethyl acetate and acetone as co-solvents gave similar results (TI-3 and TI-14). The particle size distribution (measured as the polydispersity) for all particles is considered to be broad since the polydispersity is $>0.08$ in all cases. No discernable trends can be noticed in the polydispersity data. In conclusion, particle sizes are readily controllable by alterations in the monomers and as well as changes to the reaction conditions and species concentrations. 
Table 2. Summary of thermally initiated polymerizations. All reactions used $10 \mathrm{~g}$ methanol, PVP as stabilizer, AIBN as initiator, $3 \mathrm{~h}$ at $60^{\circ} \mathrm{C}, 1: 1$ mole ratio of monomers (PETMP + TTT), and 1:1 (vol.) of co-solvent (ethyl acetate, unless otherwise stated) relative to the monomers. TI-3 is a reference reaction.

\begin{tabular}{|c|c|c|c|c|c|}
\hline \multirow{2}{*}{$\begin{array}{l}\text { Reaction } \\
\text { Name }\end{array}$} & \multicolumn{3}{|c|}{ Reactant Amounts (g) } & \multirow{2}{*}{$\begin{array}{l}\text { Avg. Particle } \\
\text { Diameter } \\
(\mathrm{nm})\end{array}$} & \multirow[t]{2}{*}{ Polydispersity } \\
\hline & Monomers & Initiator & $\begin{array}{l}\text { Stabilizer } \\
\text { (PVP) }\end{array}$ & & \\
\hline TI-1 & 0.05 & 0.005 & 0.025 & 250 & 0.200 \\
\hline TI-2 & 0.072 & 0.0071 & 0.0357 & 315 & 0.199 \\
\hline TI-3 & 0.10 & 0.01 & 0.05 & 391 & 0.126 \\
\hline TI-4 & 0.20 & 0.02 & 0.1 & 721 & 0.156 \\
\hline TI-5 & 0.30 & 0.03 & 0.15 & 752 & 0.104 \\
\hline TI-6 & 0.05 & 0.01 & 0.05 & 230 & 0.152 \\
\hline TI-7 & 0.072 & 0.01 & 0.05 & 288 & 0.162 \\
\hline TI-3 & 0.1 & 0.01 & 0.05 & 391 & 0.126 \\
\hline TI-8 & 0.2 & 0.01 & 0.05 & 755 & 0.121 \\
\hline TI-9 & 0.3 & 0.01 & 0.05 & Aggregated & \\
\hline TI-10 & 0.1 & 0.01 & 0 & Aggregated & \\
\hline TI-11 & 0.1 & 0.01 & 0.025 & 420 & 0.101 \\
\hline TI-12 & 0.1 & 0.01 & 0.1 & 290 & 0.149 \\
\hline TI-13 & 0.1 & 0.01 & 0.15 & 272 & 0.112 \\
\hline TI -3 & 0.1 & 0.01 & 0.05 & 391 & 0.126 \\
\hline TI-14 & 0.1 & 0.01 & 0.05 (acetone) & 390 & 0.100 \\
\hline
\end{tabular}




\begin{tabular}{llllll}
\hline TI-15 & 0.1 & 0.05 & 0.05 & 327 & 0.089 \\
TI-16 & 0.1 & 0.1 & 0.05 & 314 & 0.151 \\
TI-17 & 0.1 & 0.2 & 0.05 & 345 & 0.170 \\
\hline
\end{tabular}

Effect of Various Parameters on Particle Size and Distribution: Redox Initiation

The results from redox polymerizations initiated by a combination of benzoyl peroxide and a tertiary amine (ethyl 4-dimethylaminobenzoate, EDAB), are summarized in Table 3. We observed that that the redox initiator system was less efficient than both photo- and thermalinitiation. Experiments R-1 and R-2 did not form sub-micron sized particles. No phase transition occurred for R-1, and FT-IR analysis on the non-volatile mixture by concentrating from R-1 resulted in the presence of a significant amount of unreacted thiol and alkene. Increasing the organic content from $1 \mathrm{wt} . \%$ to $3 \mathrm{wt} . \%$ (R3 - R5), we observed the formation of sub-micron sized particles, although the particles did become much larger than those from the thermal and photo-initiated polymerizations. We noticed the low efficiency of redox initiation in experiments with varied initiator concentrations (R6 - R9). Contrary to previous systems where particle size decreases with initiator concentration, herein, for low loadings ( $<5 \mathrm{wt} . \%)$ of the redox initiator, the reaction conversion was low and the particles were small. In the BPO-EDAB system, radicals are generated by bimolecular reactions, and at dilute conditions the initiation could be much slower and thus lead to incomplete reactions. In the above mentioned kinetics study, a relatively large amount of redox initiator (10 wt.\% in total with respect to monomers) was used and led to complete reaction. Generally, redox initiator concentrations of up to $10 \mathrm{wt} . \%$ are required to assure full conversion. 
Table 3. Summary of redox polymerizations of PETMP and TTT in methanol with the presence of PVP. In all the reactions, $20 \mathrm{~mL}$ methanol, 1:1 mole ratio of monomers, 1:1 (vol.) of ethyl acetate relative to the monomers were used and the reaction was carried out at ambient conditions.

\begin{tabular}{|c|c|c|c|c|c|}
\hline \multirow{2}{*}{$\begin{array}{l}\text { Reaction } \\
\text { Name }\end{array}$} & \multicolumn{3}{|c|}{ Reactant Amounts (g) } & \multirow{2}{*}{$\begin{array}{l}\text { Avg. Particle } \\
\text { Diameter } \\
\text { (nm) }\end{array}$} & \multirow[t]{2}{*}{ Polydispersity } \\
\hline & Monomers & Initiator & $\begin{array}{l}\text { Stabilizer } \\
\text { (PVP) }\end{array}$ & & \\
\hline $\mathrm{R}-1$ & 0.08 & 0.008 & 0.04 & - & - \\
\hline R-2 & 0.11 & 0.011 & 0.06 & - & - \\
\hline R-3 & 0.16 & 0.016 & 0.08 & 243 & 0.142 \\
\hline $\mathrm{R}-4$ & 0.24 & 0.024 & 0.12 & 351 & 0.056 \\
\hline R-5 & 0.48 & 0.048 & 0.24 & 304 & 0.131 \\
\hline $\mathrm{R}-6$ & 0.52 & 0.005 & 0.26 & 186 & 0.058 \\
\hline R-7 & 0.52 & 0.026 & 0.26 & 354 & 0.018 \\
\hline R-8 & 0.52 & 0.105 & 0.26 & 334 & 0.126 \\
\hline R-9 & 0.52 & 0.157 & 0.26 & 803 & 0.229 \\
\hline
\end{tabular}

Various Monomers:

The choice of monomers dictates many outcomes of polymerizations, such as particle functionality, thermo-mechanical properties, and colloidal stability. Thus, we chose several other alkene, alkyne and thiol monomers to polymerize using both thermal or photo-initiation. These monomer pairs were chosen to give polymers with differing polarities, functionalities, and 
crosslink densities. The structures of the monomers are shown in Chart 1. For example, the highest crosslink density available from the thiol/ene monomers used here was obtained by using PETMP and TTT. The trithiol monomer TMTMP provides slightly lower crosslink density (when also used with TTT), while TMPEIC provides similar crosslink density to TMTMP but with higher molecular rigidity. ODY provides even higher crosslink densities when used instead of TTT, as each alkyne group reacts with two thiols. The monomers TMP, HYA and HDT all yield linear polymers or oligomers when used in combination with other difunctional monomers. While some of these systems may not produce stable particles if their $T_{g}$ is lower than ambient temperature, some diene/dithiol combinations have also been shown to yield semicrystalline polythioethers which provides particle stability.[8] The results from these monomer pairs are provided in Table 4.

Photo-initiation appeared to more reliably yield particles (e.g., M-1 - M-3 vs. M-4 - M6), and these particles are smaller than those obtained using the same monomer set but using thermal initiation (e.g., M-8 vs. M-11). A few of the polymerizations resulted in significant amounts of non-dispersed materials, indicating their colloidal instability. This behavior is dependent on the particle size, chemistry and functionality. The success of particle formation with the non-crosslinking monomer pairs (M-13 and M-14) depended on the mode of initiation largely because of the temperature difference. Thermal polymerizations did not produce particles since the linear polymers that are produced are expected to have $T_{\mathrm{m}}$ and $T_{\mathrm{g}}$ values below the reaction temperature. Hence, they would not be expected to form particles because the polymer is able to flow, and particles can adhere to one another as they grow. Furthermore, neither reactions M-13 or M-14 produced particles when the monomer content was 1 wt.\%, but increasing to $3 \mathrm{wt} . \%$ resulted in particles when photoinitiated. 
Table 4. Summary of Polymerizations using Various Monomers. ${ }^{a}$

\begin{tabular}{|c|c|c|c|c|c|}
\hline Reaction & Ene (or Yne) & Thiol & Initiation & Avg. Particle & Polydispersity \\
\hline Name & Monomer & Monomer & Type & $\begin{array}{l}\text { Diameter } \\
(\mathbf{n m})\end{array}$ & \\
\hline M-1 & TTT & TMTMP & Photo & 312 & 0.118 \\
\hline M-2 & TTT & HDT & Photo & 570 & 0.132 \\
\hline M-3 & TTT & TMPEIC & Photo & 250 & 0.123 \\
\hline$M-4^{b}$ & TTT & TMTMP & Thermal & 740 & 0.024 \\
\hline$M-5^{b}$ & TTT & HDT & Thermal & 500 & 0.037 \\
\hline M- $6^{\mathrm{b}}$ & TTT & TMPEIC & Thermal & 367 & 0.024 \\
\hline M-7 & TMP & PETMP & Photo & 321 & 0.204 \\
\hline M-8 & ODY & PETMP & Photo & 191 & 0.194 \\
\hline M-9 & HYA & PETMP & Photo & 434 & 0.077 \\
\hline$M-10^{b}$ & TMP & PETMP & Thermal & 643 & 0.024 \\
\hline M-11 & ODY & PETMP & Thermal & 386 & 0.238 \\
\hline M-12 & HYA & PETMP & Thermal & 715 & 0.129 \\
\hline$M-13^{c}$ & TMP & HDT & Photo & 486 & 0.005 \\
\hline$M-14^{c}$ & TMP & HDT & Thermal & $\begin{array}{l}\text { Oligomers } \\
\text { only }\end{array}$ & \\
\hline
\end{tabular}

a) All reactions used $10 \mathrm{~g}$ methanol, $0.05 \mathrm{~g}$ PVP as stabilizer, 1:1 mole ratio of monomer functionality, 1 wt.\% monomers (relative to methanol), and 1:1 (vol.) of co-solvent (ethyl acetate). Photoinitiated polymerizations used $0.01 \mathrm{~g} 1$-hydroxycyclohexyl phenyl ketone as initiator and were irradiated for 5 minutes. Thermally initiated polymerizations used $0.01 \mathrm{~g}$ 
AIBN and were heated at $60^{\circ} \mathrm{C}$ for 3 hours (unless otherwise noted). b) Reactions had material settle out (TTT/TMTMP 92 wt.\%; TTT/DHT 15 wt.\%; TTT/TEMPIC 20 wt.\%; TMP/PETMP 39 wt.\%) c) Used 3 wt.\% monomers.

\section{Conclusions}

We report here that radical thiol-ene and thiol-yne 'click' polymerizations are readily used in dispersion polymerizations in alcoholic solvent systems. These polymerizations and the subsequent particle formation can be initiated using any of thermal, photo- or redox methods, depending on the specific monomer-solvent system chosen and the conditions. The particles obtained had diameters that were typically several hundred nanometers, and their size distributions were broad. Photo-initiation yielded the smallest sizes due to the rapid nucleation of particles. This additional approach to particle formation using the facile radical thiol-ene and thiol-yne polymerizations can now be added to the toolbox for making polymer colloids/microparticles.

\section{Acknowledgments}

The authors acknowledge funding from the National Science Foundation (NSF Grant No. DMR 1310528), and thank Kelly R. Tillman for technical assistance. 


\section{References}

[1] O.Z. Durham, S. Krishnan, D.A. Shipp, Polymer Microspheres Prepared by Water-Borne Thiol-Ene Suspension Photopolymerization, ACS Macro Lett. 1 (2012) 1134-1137.

[2] O.Z. Durham, D.A. Shipp, Suspension thiol-ene photopolymerization: Effect of stabilizing agents on particle size and stability Polymer 55 (2014) 1674-1680.

[3] O.Z. Durham, H.R. Norton, D.A. Shipp, Functional Polymer Particles via Thiol-Ene and Thiol-Yne Suspension "Click" Polymerization, RSC Adv. 5 (2015) 66757-66766.

[4] O.Z. Durham, D.A. Shipp, Suspension "click" polymerizations: thiol-ene polymer particles prepared with natural gum stabilizers, Colloid Polym. Sci. 293 (2015) 23852394.

[5] C. Wang, S. Chantani, M. Podgórski, C.N. Bowman, Thiol-Michael Addition Miniemulsion Polymerizations: Functional Nanoparticles and Reactive Latex Films, Polym. Chem. 6 (2015) 3758-3763.

[6] C. Wang, M. Podgórski, C.N. Bowman, Monodisperse functional microspheres from step-growth "click" polymerizations: Preparation, functionalization and implementation, Mater. Horiz. 1 (2014) 535-539.

[7] C. Wang, X.P. Zhang, M. Podgórski, W.X. Xi, J. Stansbury, C.N. Bowman, Monodispersity/Narrow Polydispersity Cross-Linked Microparticles Prepared by StepGrowth Thiol-Michael Addition Dispersion Polymerizations, Macromolecules 48 (2015) 8461-8470.

[8] F. Jasinski, E. Lobry, B. Tarablsi, A. Chemtob, C. Croutxe-Barghorn, D. Le Nouen, A. Criqui, Light-mediated thiol-ene polymerization in miniemulsion: A fast route to semicrystalline polysulfide nanoparticles, ACS Macro Lett. 3 (2014) 958-962. 
[9] J.J. Tan, C.M. Li, H. Li, H. Zhang, J.W. Gu, B.L. Zhang, Q.Y. Zhang, Water-Borne Thiol-Isocyanate Click Chemistry in Microfluidics: Rapid and Energy-Efficient Preparation of Uniform Particles, Polym. Chem. 6 (2015) 4366-4373.

[10] J.J. Tan, C.M. Li, J. Zhou, C.J. Yin, B.L. Zhang, J.W. Gu, Q.Y. Zhang, Fast and Facile Fabrication of Porous Polymer Particles via Thiol-Ene Suspension Photopolymerization, RSC Adv. 4 (2014) 13334-13339.

[11] D.N. Amato, D.V. Amato, B.R. Donovan, J.R. Douglas, S.E. Walley, A.S. Flynt, D.L. Patton, Functional, composite polythioether nanoparticles via thiol-alkyne photopolymerization in miniemulsion, Chem. Commun. 51 (2015) 10910-10913.

[12] D.V. Amato, D.N. Amato, A.S. Flynt, D.L. Patton, Functional, Sub-100 nm Polymer Nanoparticles via Thiol-Ene Miniemulsion Photopolymerization, Polym. Chem. 6 (2015) $5625-5632$.

[13] D.N. Amato, D.V. Amato, O.V. Mavrodi, D.A. Braasch, S.E. Walley, J.R. Douglas, D.V. Mavrodi, D.L. Patton, Destruction of Opportunistic Pathogens via Polymer NanoparticleMediated Release of Plant-Based Antimicrobial Payloads, Adv. Healthcare Mater. 5 (2016) 1094-1103.

[14] F. Jasinski, A. Ranneé, J. Schweitzer, D. Fischer, E. Lobry, C. Croutxe-Barghorn, M. Schmutz, D. LeNouen, A. Criqui, A. Chemtob, Thiol-Ene Linear Step-Growth Photopolymerization in Miniemulsion: Fast Rates, Redox-Responsive Particles, and Semicrystalline Films, Macromolecules 49 (2016) 1143-1153.

[15] P. Espeel, F.E. DuPrez, “Click”-Inspired Chemistry in Macromolecular Science: Matching Recent Progress and User Expectations, Macromolecules 48 (2015) 2-14. 
[16] R. Hoogenboom, Thiol-yne chemistry: A powerful tool for creating highly functional materials, Angew. Chem. Int. Ed. 49 (2010) 3415-3417.

[17] C.E. Hoyle, C.N. Bowman, Thiol-Ene Click Chemistry, Angew. Chem. Int. Ed. 49 (2010) 1540-1573.

[18] C.E. Hoyle, A.B. Lowe, C.N. Bowman, Thiol-click chemistry: A multifaceted toolbox for small molecule and polymer synthesis, Chem. Soc. Rev. 39 (2010) 1355-1387.

[19] A.B. Lowe, C.E. Hoyle, C.N. Bowman, Thiol-yne click chemistry: A powerful and versatile methodology for materials synthesis, J. Mater. Chem. 20 (2010) 4745-4750.

[20] C.E. Hoyle, T.Y. Lee, T. Roper, Thiol-Enes: Chemistry of the Past with Promise for the Future, J. Polym. Sci. Part A: Polym. Chem. 42 (2004) 5301-5338.

[21] R.A. Prasath, M.T. Gokmen, P. Espeel, F.E. DuPrez, Thiol-ene and thiol-yne chemistry in microfluidics: a straighforward method towards macroporous and nonporous functional polymer beads, Polym. Chem. 1 (2010) 685-692.

[22] C.O. Bounds, R. Goetter, J. Pojman, M. Vandersall, Preparation and application of microparticles prepared via the primary amine-catalyzed Michael addition of a trithiol to a triacrylate, J. Polym. Sci. Part A: Polym. Chem. 50 (2012) 409-422.

[23] R. Arshady, Suspension, emulsion, and dispersion polymerization: A methodological survey, Colloid Polym. Sci. 270 (1992) 717-732.

[24] K. Landfester, Miniemulsion Polymerization and the Structure of Polymer and Hydrid Nanoparticles, Angew. Chem. Int. Ed. 48 (2009).

[25] K. Landfester, A. Musyanovych, V. Mailänder, From Polymeric Particles to Multifunctional Nanocapsules for Biomedical Applications Using the Miniemulsion Process, J. Polym. Sci. Part A: Polym. Chem. 48 (2010) 493-515. 
[26] M. Barrère, K. Landfester, High molecular weight polyurethane and polymer hybrid particles in aqueous miniemulsion, Macromolecules 36 (2003) 5119-5125.

[27] K. Landfester, F. Tiarks, H.-P. Hentze, M. Antonietti, Polyaddition in miniemulsions: A new route to polymer dispersions, Macromol. Chem. Phys. 201 (2000) 1-5.

[28] M. Barrère, K. Landfester, Polyester synthesis in aqueous miniemulsion, Polymer 44 (2003) 2833-2841.

[29] J. Pecher, S. Mecking, Nanoparticles from step-growth coordination polymerization, Macromolecules 40 (2007) 7733-7735.

[30] F.M. Bauers, R. Thomann, S. Mecking, Submicron polyethylene particles from catalytic emulsion polymerization, J. Am. Chem. Soc. 125 (2003) 8838-8840.

[31] P.B. Cardoso, A. Musyanovych, K. Landfester, C. Sayer, P.H.H. deAraújo, M.A.R. Meier, ADMET Reactions in Miniemulsion, J. Polym. Sci. Part A: Polym. Chem. 52 (2014).

[32] S. Mecking, Polymer dispersions from catalytic polymerization in aqueous systems, Colloid Polym. Sci. 285 (2007) 605-619.

[33] S. Mecking, A. Held, F.M. Bauers, Aqueous Catalytic Polymerization of Olefins, Angew. Chem. Int. Ed. 41 (2002) 544-561.

[34] A.J. Paine, W. Luymes, J. McNulty, Dispersion Polymerization of Styrene in Polar Solvents. 6. Influence of Reaction Parameters on Particle Size and Molecular Weight in Poly(N-vinylpyrrolidone)-Stabilized Reactions, Macromolecules 23 (1990) 3104-3109. 
Graphical Abstract:

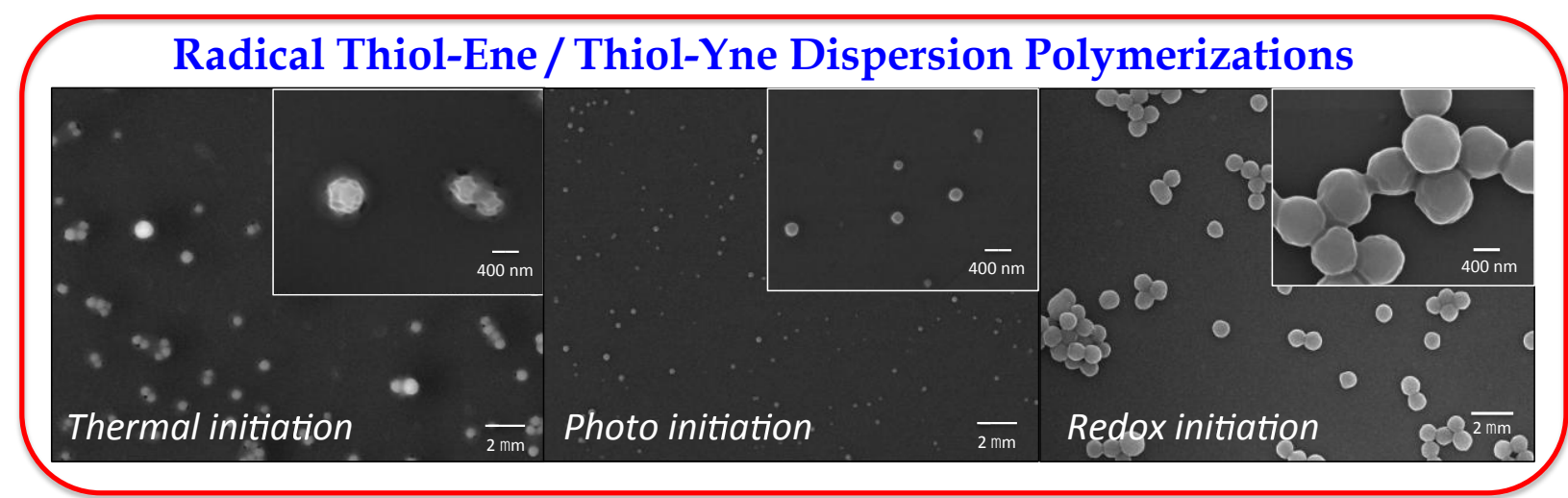

